\title{
Injection to the pick-up ion regime from high energies and induced ion power-laws
}

\author{
H.-J. Fahr ${ }^{1}$, I. V. Chashei ${ }^{2}$, and D. Verscharen ${ }^{1,3}$
}

\author{
1 Argelander Institute for Astronomy, University of Bonn, Auf dem Hügel 71, 53121 Bonn, Germany \\ 2 Lebedev Physical Institute, Leninskii pr. 53, 117924 Moscow, Russia \\ 3 Max Planck Institute for Solar System Research, Max-Planck-Str. 2, 37191 Katlenburg-Lindau, Germany \\ e-mail: dverscha@astro.uni-bonn.de
}

Received 6 August 2008 / Accepted 29 July 2009

\begin{abstract}
Though pick-up ions (PUIs) are a well-known phenomenon in the inner heliosphere, their phase-space distribution nevertheless is a theoretically unsettled problem. Especially the question of how PUIs form their suprathermal tails, extending to far above their injection energies, still now is unsatisfactorily answered. Though Fermi-2 velocity diffusion theories have revealed that such tails are populated, they nevertheless show that resulting population densities are much less than seen in observations showing power-laws with a velocity index of " -5 ". We first investigate here, whether or not observationally suggested power-laws can be the result of a quasi-equilibrium state between suprathermal ions and magnetohydrodynamic turbulences in energy exchange with each other. We demonstrate that such an equilibrium cannot be established, since it would require too high PUI pressures enforcing a shock-free deceleration of the solar wind. We furthermore show that Fermi-2 type energy diffusion in the outer heliosphere is too inefficient to determine the shape of the distribution function there. As we can show, however, power-laws beyond the injection threshold can be established, if the injection takes place at higher energies of the order of $100 \mathrm{keV}$. As we demonstrate here, such an injection is connected with modulated anomalous cosmic ray (ACR) particles at the lower end of their spectrum when they again start being convected outwards with the solar wind. Therefore, we refer to these particles as ACR-PUIs. In our quantitative calculation of the PUI spectrum resulting under such conditions we in fact find again power-laws, however with a velocity-power index of " -4 " and fairly distance-independent spectral intensities. As it seems these facts are observationally well supported by VOYAGER measurements in the lowest energy channels.
\end{abstract}

Key words. plasmas - solar wind - cosmic rays

\section{Introduction}

Suprathermal ions, picked up by the supersonic solar wind flow as ionized neutral atoms, have become known as pick-up ions (PUIs) and are produced all over the inner heliosphere with a typical upwind-downwind asymmetry with respect to the inflow direction of the neutral ISM inflow vector (Rucinski et al. 1993; Fahr \& Rucinski 1999). In the case of PUI protons, their production is due to photoionization and charge exchange of interstellar H-atoms (see Rucinski \& Fahr 1991; Fahr \& Rucinski 1999; Rucinski et al. 2003; Bzowski et al. 2008). Their spatial distribution seems well understood, while the PUI-phase-space transport is a much less settled subject. Especially it exists an ongoing debate of how efficiently PUIs just after the pick-up process are accelerated to higher energies due to nonlinear wave-particle interactions (see e.g. Isenberg 1987; Bogdan et al. 1991; Fichtner et al. 1996; Chalov \& Fahr 1996, 1998; Fichtner 2001; Chalov et al. 2004) and whether at all energy diffusion plays a relevant role in this transport.

Some hint is given by the solar wind proton temperature behavior with distance. The observed non-adiabatic temperature behavior namely proves that a specific solar wind proton heating must operate in the outer heliosphere which can only be due to energy absorption from PUI generated turbulence, since convected turbulence amplitudes quickly die out with distance (see Smith et al. 2001; Fahr \& Chashei 2002a).
Freshly injected PUIs represent keV-energetic protons in the supersonic solar wind frame and may be called here: "primary pick-up ions" (or: PUIs*). The velocity distribution of these newly produced PUIs* is toroidal and unstable (see Winske \& Leroy 1984; Winske et al. 1985; Lee \& Ip 1987; Fahr \& Ziemkiewicz 1988). With the free energy of this unstable distribution PUIs* drive Alfvénic wave power. The latter enforces pitch-angle isotropization of the initial velocity distribution (see Chalov \& Fahr 1998, 1999a). Due to wave-wave coupling, the wave energy generated by PUIs* at the injection wavelength $\lambda_{\mathrm{i}}=U_{\mathrm{s}} / \Omega_{\mathrm{p}}$ is diffusively transported in wavevector space both to smaller wavelengths where it can be absorbed by solar wind protons and to larger wavelengths where it is reabsorbed by all PUIs. This effect is seen as the main reason of solar wind proton heating occurring in the outer heliosphere (Smith et al. 2001; Fahr \& Chashei 2002b; Chashei et al. 2003; Stawicki 2004). Only a small fraction of about $5 \%$ of the PUI-generated wave energy reappears in the observed proton temperatures. Freshly injected PUIs excite turbulences that can organize a power-law distribution. From this distribution, both the solar wind ions and the PUIs themselves can absorb energy as shown by Chashei et al. (2003). Also the approach by Isenberg et al. (2003) where energy diffusion of PUIs is not taken into account shows that only a low degree (2-5\%) of the PUI driven wave energy is absorbed by solar wind protons in form of thermal energy. This raises the question where the major portion of the wave 
energy produced during the primary pick-up process goes to. To clarify the energy redistributions, kinetic and spectral details of the relevant processes have to be investigated. A detailed numerical study of the PUI velocity distribution and the spectral Alfvénic/Magnetosonic wave power evolution has meanwhile been carried out (Chalov et al. 2004, 2006b) and presents a simultaneous solution of a coupled system of equations consistently describing the isotropic velocity distribution function of PUIs and the spectral wave power intensity.

As one can see from this study, the largest portion of the self-generated wave energy is reabsorbed by PUIs themselves as a result of the cyclotron resonant interaction and leads to PUIacceleration. It could perhaps be hoped that this energization of pick-up protons due to Fermi-2 stochastic acceleration processes eventually leads to the ubiquitous power-law PUI-tails pointed out by Fisk \& Gloeckler $(2006,2007)$. To the opposite, however, as reflected in the results presented by Chalov et al. (2004, 2006b) it is evident that this is not the case: even at larger distances close to the termination shock (100 AU) the PUI distributions show a rapid cut-off at energies higher than the injection energy. The question thus is raised here why power-laws have been seen at all. An explanation that we are favoring here is a new injection source to the PUI regime from high energies connected with modulated anomalous cosmic ray (ACR) particles. These protons are primary ACR particles that occur with a spectrum down to the typical energy of the usually assumed PUIs. At this part of the spectrum, both particle species cannot be distinguished. Therefore, we refer to them as ACR-PUIs.

In Sect. 2, we investigate the physical possibility of powerlaw ions in the outer heliosphere as they are recently proposed by several authors and we find that they cannot occur with a power-index of -5 . As we show in Sect. 3, the proposed processes are not effective enough to produce the desired ion tails which means that another mechanism has to lead to the observed spectrum. In Sect. 4, we show how a high-energy source can be derived by taking a modulated ACR spectrum upstream of the solar wind termination shock. This injection mechanism is discussed in Sect. 5 where we show that these high-energy ions can lead to power-law ion tails, however with a power-index of -4 . The results are discussed and compared with observations in Sect. 6.

\section{Can power-law ion distributions be in equilibrium with hydromagnetic turbulence?}

Challenged by recent results concerning ion spectra at large distances (McDonald et al. 2003; Decker et al. 2005; Király 2005; Fisk \& Gloeckler 2006), we look into the problem of PUI-phasespace transport under these new given auspices. First we discuss the argument given by Fisk \& Gloeckler $(2006,2007)$ that PUIs under resonant interaction with ambient compressive turbulences enter a quasi-equilibrium state with saturated powerlaw distributions of a somehow sacrosanct spectral velocity index of $\gamma_{v}=-5$.

The related, well-known Kolmogorov formalism is based on a "dimensional" reasoning: The problem concerning the energy distribution in eddies of a typical wave number $k$ is considered using two different dimensional quantities: namely the spectral energy density $E_{k}$ and the wave number $k$. The spectral energy flux is then defined by

$S=\left(k E_{k}\right) / \tau_{k}$ and has to be constant for stationary cases. $\tau_{k}$ is the typical life time of eddies with scale $\lambda_{k}=2 \pi / k$ and is given by

$\tau_{k}=\left(k v_{k}\right)^{-1}$

with the typical vortex velocity $v_{k}$ given by

$v_{k}=\left(E_{k} k\right)^{1 / 2}$.

Requiring a constant energy flux $S$ given by Eq. (1), with $\tau_{k}$ given by Eq. (2) then automatically leads to the Kolmogorov spectrum in the form $E_{k} \sim k^{-5 / 3}$.

In their thermodynamical approach to particle spectra in equilibrium with waves, Fisk \& Gloeckler (2006, 2007), however, only consider one single-dimensional quantity, i.e. the kinetic energy $T$, because in their case the energy distribution $F$ has the dimension $[F]=[T]^{-1}$. The flux combination

$S_{F}=T \cdot F \cdot \frac{\Delta T}{\Delta t}$

is then expected by them to be constant and, thus, the energy gain is given by

$\frac{\Delta T}{\Delta t} \sim T$

and hence the above requirements result in the requirement that $F \sim T^{-2}$.

This shows that the analogy of the Fisk-Gloeckler approach with the Kolmogorov formalism is not complete, since in their theory the quantity $\Delta t$ is not specified. Only if we play the same game with the Kolmogorov turbulence using instead of Eq. (2) the assumption $\tau=(k u)^{-1}$, where $u$ is some independent external speed (especially independent of $k$ ), we will find from Eq. (1) also the result

$E_{k} \sim k^{-2}$

analogous to the result obtained by Fisk-Gloeckler.

The problem may be briefly inspected here whether or not some spatial/temporal disturbances in the solar wind plasma can be considered as waves. They should be considered as waves, if the convection time $\tau_{\text {conv }}=r / U$ is much greater than the passage period of these waves in the wind reference frame $\tau_{\sim}=2 \pi /\left(k_{0} v_{\mathrm{A}}\right)$ where $r$ denotes the radial distance to the sun and $U$ the solar wind bulk velocity. The Alfvén speed is designated as $v_{\mathrm{A}}$ with the corresponding wavevector $k_{0}$ for the turbulence. For an inequality of the kind $\tau_{\sim} \ll t_{\text {conv }}$, this then leads to the definition of the principal wave turbulence correlation scale given by $L_{\mathrm{m}}<r v_{\mathrm{A}} / U \approx 0.1 \mathrm{AU}$. A more exact definition of the turbulence correlation scale is given in the paper of Chashei et al. (2003). This value is also in accordance with the value used by Fahr (2007) for his estimation of the upper possible velocity border. Some measurements indicate smaller values for the turbulence correlation length than calculated by us in this paper (see e.g. Matthaeus et al. 2005). However, the following conclusions in our paper drawn on the effects of turbulent heating of suprathermal protons become even less promising for an MHD-equilibrium to be established, if smaller correlation lengths prevail. One can expect that at larger distances, $r \geq$ $1 \mathrm{AU}$, the value for $L_{\mathrm{m}}$ increases proportionally to $r$, since a similar dependence is also valid for the outer scale of turbulence $k_{0}^{-1}$ (see Chashei et al. 2003). Here we estimate the maximum energy for protons resonating with these largest scales $L_{\mathrm{m}}$ at distances of about $100 \mathrm{AU}$ and find with $v_{\max } \simeq \Omega(100 \mathrm{AU})$. $L_{\mathrm{m}}(100 \mathrm{AU}) / \gamma\left(v_{\max }\right) \simeq \Omega(1 \mathrm{AU}) \cdot L_{\mathrm{m}}(1 \mathrm{AU}) / \gamma\left(v_{\max }\right)$, where 
$v_{\max }, \gamma\left(v_{\max }\right)$ and $\Omega$ are the maximal ion speed, the associated Lorentz factor and the ion gyrofrequency, respectively. Protons with these speeds have energies of about $E_{\max } \approx 1 \mathrm{GeV}$ independent of distance. Thus, ions with $E \leq E_{\max }$ can be expected to be scattered by waves, whereas ions with $E \geq E_{\max }$ can only be scattered by large scale velocity structures in the solar wind.

Since in any case, however, power-laws seem to be a fact well supported by observations within a certain range of solar distances and ion energies, we shall nevertheless take this finding as serious here as deserved and determine in the following the absolute spectral intensity of this PUI-power-law distribution and its consequences.

If the PUI distribution is given in the form

$f_{\text {pui }}(r, v)=f_{\text {pui }, 0} \cdot\left(\frac{v}{v_{0}}\right)^{\gamma_{v}}$

with the velocity-power index $\gamma_{v}=-5$, then the PUI density is given by

$n_{\text {pui }}(r)=4 \pi f_{\text {pui, }, 0} \cdot \int_{v_{0}}^{v_{\infty}}\left(\frac{v}{v_{0}}\right)^{-5} v^{2} \mathrm{~d} v \simeq 2 \pi f_{\text {pui }, 0} \cdot v_{0}^{3}$,

where $f_{\text {pui, } 0}=f_{\text {pui, } 0}(r)$ is a local normalization value, and $v_{0}$ and $v_{\infty}$ are lower and upper velocity limits of the quasistationary PUI-power-law.

Using $\psi=v / v_{0}$, one obtains for the power-law PUI pressure as the second moment of the distribution function the following result:

$P_{\text {pui }}(r)=4 \pi f_{\text {pui }, 0} \frac{m}{2} v_{0}^{5} \int_{1}^{\psi_{\infty}} \psi^{\prime-5} \psi^{\prime 4} \mathrm{~d} \psi^{\prime}=2 \pi m \cdot f_{\text {pui }, 0} v_{0}^{5} \ln \left(\psi_{\infty}\right)$.

Evidently, the definition of $P_{\text {pui }}(r)$ requires the determination of all three local values $f_{\text {pui, },}(r), v_{0}(r)$, and $v_{\infty}(r)$ which we aim at below.

\subsection{Determination of the inner and outer velocity border}

Using the definition of the lower velocity border as done by Fahr (2007) one finds

$v_{0} \simeq \frac{1}{5} U\left(\frac{r}{r_{\mathrm{E}}}\right)^{1 / 4}$. form

With the above result one obtains the PUI distribution in the

$$
f_{\text {pui }, 0}=n_{\text {pui }}(r) \cdot \frac{1}{2 \pi v_{0}^{3}}=\frac{5^{3}}{2 \pi U^{3}} n_{\text {pui }}(r) \cdot\left(\frac{r}{r_{\mathrm{E}}}\right)^{-3 / 4} .
$$

The local PUI density can be derived from the interplanetary $\mathrm{H}$-atom density $n_{\mathrm{H}}(r, \theta)$, which depends on the radial distance $r$ and the inclination angle $\theta$ with respect to the upwind axis, and the effective (charge exchange + photoionization)-induced injection rate $\beta_{\text {pui }}=n_{\mathrm{H}}(r, \theta)\left[n_{\mathrm{s}}(r) \sigma_{\mathrm{ex}} U+v_{\mathrm{i}}\right]$. Here $n_{\mathrm{s}}(r), \sigma_{\mathrm{ex}}$, $U, v_{\mathrm{i}}$ denote the solar wind proton density, the charge exchange cross section, the solar wind bulk velocity and the photoionization frequency. The $\mathrm{H}$-atom density at distances $r \geq 5 \mathrm{AU}$ in the upwind hemisphere is satisfactorily well given by the following expression (see Fahr 1971):

$n_{\mathrm{H}}(r, \theta)=n_{\mathrm{H}, \infty} \exp \left[-\frac{\beta_{\text {pui }, 0} r_{0}^{2} \theta}{U r \sin \theta}\right]$ and thus leads to the following PUI density (also see Fahr \& Rucinski 1999)

$n_{\text {pui }}(r, \theta)=\left(\frac{r_{0}}{r}\right)^{2} n_{\text {pui }, 0}+\frac{1}{r^{2} U} \int_{r_{0}}^{r} \beta_{\text {pui }}\left(r^{\prime}, \theta\right) r^{\prime 2} \mathrm{~d} r^{\prime}$.

From the above one derives the following radial space derivative which later on in this paper will be needed:

$\frac{\partial n_{\mathrm{pui}}(r, \theta)}{\partial r}=\frac{-2 n_{\mathrm{pui}}(r, \theta)}{r}+\frac{\beta_{\mathrm{pui}}(r, \theta)}{U}$

To calculate the PUI pressure, one more quantity in addition is needed, namely the upper velocity border $v_{\infty}$. To determine $v_{\infty}$ we follow the idea presented by Fisk \& Gloeckler (2006, 2007) assuming that PUI-power-law distributions result from a specific quasi-equilibrium state self-establishing such that the wave field transfers per unit of time as much energy to PUIs by energy diffusion, as energy is expended in the solar wind frame for the work done by the pressure gradient of comoving PUIs against the magnetosonic fluctuations. The important restriction to energy diffusion by nonlinear interaction with the compressive fluctuations is that the typical diffusion period $\tau_{\text {diff }} \simeq 3 L_{\mathrm{m}}^{2} / v \lambda_{\|}$should be much larger than the convection period given by $\tau_{\text {conv }} \simeq L_{\mathrm{m}} / U$ (see Chalov et al. 2003). This leads to the requirement

$L_{\mathrm{m}}>\frac{v \lambda_{\|}}{3 U}$

with $\lambda_{\|}$as the mean free path for particles parallel to the magnetic field. The uppermost velocity $v_{\infty}$ is the limit at which this condition is just fulfilled:

$v_{\infty} \simeq \frac{3 U L_{\mathrm{m}}}{\lambda_{\|}}$

We assume the interaction of the particles with a slab Alfvénic turbulence field. The mean free path is given by

$\lambda_{\|}=\frac{3 v}{8} \int_{-1}^{+1} \frac{\left(1-\mu^{2}\right)^{2}}{D_{\mu \mu}} \mathrm{d} \mu$

with the pitch-angle diffusion coefficient

$D_{\mu \mu}=D_{v v, 0} v_{\mathrm{A}}^{-2}\left(\frac{U^{3}}{r_{\mathrm{E}}}\right)\left(\frac{v}{U}\right)\left(\frac{r_{\mathrm{E}}}{r}\right)^{3 / 4}$

from Chalov et al. (2003) for cyclotron resonant wave-particle interaction with unpolarized, one-dimensional, and isotropic turbulence, which leads to the velocity-independent expression

$\lambda_{\|}=\frac{2}{5} v_{\mathrm{A}}^{2}\left(\frac{r_{\mathrm{E}}}{U^{3}}\right)\left(\frac{U}{D_{v v, 0}}\right)\left(\frac{r_{\mathrm{E}}}{r}\right)^{3 / 4}=\lambda_{\|, \mathrm{E}}\left(\frac{r_{\mathrm{E}}}{r}\right)^{3 / 4}$

for the mean free path, with the reference value

$D_{v v, 0}=\frac{\sqrt{\left\langle\delta u^{2}\right\rangle_{\mathrm{E}}}}{U} \frac{r_{\mathrm{E}}}{9 L_{\mathrm{m}}}$

for the diffusion coefficient. Therefore, the upper velocity border is given by

$v_{\infty} \simeq \frac{3 U L_{\mathrm{m}}}{\lambda_{\|, \mathrm{E}}}$, 
which evaluates to

$v_{\infty} \simeq \frac{9}{0.3}\left(\frac{r_{\mathrm{E}}}{r}\right)^{3 / 4} U$

by taking $L_{\mathrm{m}}=3 \mathrm{AU}$ and $\lambda_{\|, \mathrm{E}}=0.3 \mathrm{AU}$ (Chalov \& Fahr 1999b). The ratio

$\psi_{\infty}=\frac{v_{\infty}}{v_{0}}=150 x^{-1}$

is needed in the later calculation and should be compared with the result obtained by Fahr (2007) deriving the upper velocity border from the study of the uppermost resonance possibilities of ions with the largest prevailing correlation lengths $L_{\mathrm{m}}$ existing in the solar wind velocity structures, yielding the result

$\psi_{\infty}=30 \cdot x^{-3 / 4}$,

which gives smaller values than those derived for conditions when balanced pressure equilibrium in the sense of Fisk \& Gloeckler (2007) is adopted. Here below we shall demonstrate that this value for $\psi_{\infty}$, which is the direct consequence of the assumptions by Fisk, definitely leads to unreasonable consequences when we investigate the associated PUI pressure.

\subsection{The upstream PUI pressure}

We calculate the PUI pressure resulting from power-law distributed PUIs upstream of the shock and find with Eqs. (9)-(11) and (23)

$P_{\text {pui }}(x)=m U^{2} n_{\text {pui }}(x) x^{1 / 2} \ln \left(150 x^{-1}\right)$,

where we have introduced $x=r / r_{\mathrm{E}}$. We obtain the pressure gradient by differentiation:

$$
\begin{aligned}
\frac{\partial P_{\text {pui }}}{\partial x}= & m U^{2} \times\left[\frac{\partial n_{\text {pui }}}{\partial x} x^{1 / 2} \ln \left(150 x^{-1}\right)\right. \\
& \left.+\frac{1}{2} n_{\text {pui }} x^{-1 / 2} \ln \left(150 x^{-1}\right)-n_{\text {pui }} x^{-1 / 2}\right],
\end{aligned}
$$

which leads with Eq. (14) to

$$
\begin{aligned}
\frac{\partial P_{\text {pui }}}{\partial x}= & m U^{2} \times\left[-\frac{3}{2} n_{\text {pui }} x^{-1 / 2} \ln \left(150 x^{-1}\right)\right. \\
& \left.+\frac{\beta r_{\mathrm{E}}}{U} x^{1 / 2} \ln \left(150 x^{-1}\right)-n_{\text {pui }} x^{-1 / 2}\right] .
\end{aligned}
$$

If now we derive the effective upstream Mach number, neglecting thereby solar wind electron and proton pressures compared to the PUI pressure, i.e. assuming $P_{\mathrm{e}} ; P_{\mathrm{s}} \ll P_{\text {pui }}$, and following the definitions by Fahr \& Rucinski (1999), we obtain

$$
\begin{aligned}
M_{\mathrm{s}}^{* 2} & =\left(\frac{U}{C_{\mathrm{s}}^{*}}\right)^{2}=\frac{U^{2} \frac{\partial \rho_{\mathrm{s}}}{\partial r}}{\frac{\partial}{\partial r}\left(P_{\mathrm{s}}+P_{\mathrm{e}}+P_{\mathrm{pui}}\right)} \\
& \simeq \frac{2 n_{\mathrm{s}}}{\frac{3}{2} n_{\text {pui }} x^{1 / 2} \ln \left(150 x^{-1}\right)-\frac{\beta r_{\mathrm{E}}}{U} x^{3 / 2} \ln \left(150 x^{-1}\right)+n_{\text {pui }} x^{1 / 2}} .
\end{aligned}
$$

With $\Lambda=\frac{\beta r_{0}}{U n_{\mathrm{s}}} \simeq 10^{-2}$ and the PUI abundance $\xi=n_{\text {pui }} / n_{\mathrm{s}} \simeq 0.2$ (see Fahr \& Rucinski 1999), we obtain

$$
M_{\mathrm{S}}^{* 2}=\frac{2 x^{-1 / 2}}{\xi\left(\frac{3}{2} \ln \left(150 x^{-1}\right)+1\right)-\Lambda \frac{r_{\mathrm{E}}}{r_{0}} x \ln \left(150 x^{-1}\right)} .
$$

For a shock position at $x \simeq 100$ and with the lower reference distance $r_{0} \simeq 5 \mathrm{AU}$, this expression yields

$M_{\mathrm{s}}^{* 2}=0.83$

which means that the effective upstream Mach number $M_{\mathrm{s}}^{*}=$ 0.91 is lower than 1 .

Hence, power-law PUI pressures in balance with the wave fields would not allow for the occurrence of a termination shock of the solar wind (i.e. needing upstream Mach numbers $M_{\mathrm{s}}^{*} \geq 1$ !).

The non-existence of the above "turbulence-particle"equilibrium (TPE) can also be concluded along a different line of argumentations together with the calculation of the downstream PUI pressure connected with power-law distributed PUIs. Using the results presented in Fahr \& Lay (2000), one obtains on the basis of Liouville's theorem and conservation of magnetic moment at the passage from upstream to downstream over the shock the PUI-distribution function downstream of the shock (indicated by index 2) by the following relation:

$f_{\text {pui, } 2}(v)=\frac{1}{\sqrt{s}} f_{\text {pui }, 1}\left(\frac{v}{\sqrt{s}}\right)$

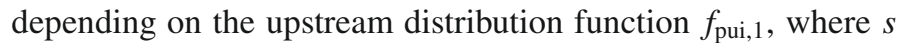
denotes the compression ratio at the shock. From that relation one obtains for the downstream PUI pressure $P_{\text {pui,2 }}$ the following expression

$$
\begin{aligned}
P_{\text {pui }, 2} & =\frac{m}{2 \sqrt{s}} \int_{\sqrt{s} v_{0}}^{\sqrt{s} v_{\infty}} f_{\text {pui }, 1}(v / \sqrt{s}) v^{4} \mathrm{~d} v \\
& =\frac{m}{2} s^{2} \int_{v_{0}}^{v_{\infty}} f_{\text {pui }, 1}(v) v^{4} \mathrm{~d} v=s^{2} P_{\text {pui }, 1},
\end{aligned}
$$

which states that the downstream PUI pressure is enhanced with respect to the upstream PUI pressure by the factor $s^{2}$. Reminding that the latter pressure for TPE-conditions is given by

$P_{\text {pui, } 1}\left(r_{\mathrm{sh}}\right)=m n_{\text {pui }}\left(r_{\mathrm{sh}}\right) U^{2}\left(\frac{r_{\mathrm{sh}}}{r_{\mathrm{E}}}\right)^{1 / 2} \ln \left(150 \frac{r_{\mathrm{E}}}{r_{\mathrm{sh}}}\right)$

one finds that the downstream PUI pressure should amount to

$P_{\text {pui, } 2}\left(r_{\mathrm{sh}}\right)=m n_{\text {pui }}\left(r_{\mathrm{sh}}\right) s^{2} U^{2}\left(\frac{r_{\mathrm{sh}}}{r_{\mathrm{E}}}\right)^{1 / 2} \ln \left(150 \frac{r_{\mathrm{E}}}{r_{\mathrm{sh}}}\right)$,

which normalized with the upstream solar wind kinetic energy density $\epsilon_{\text {kin }}=(1 / 2) m U^{2}\left(n_{\mathrm{s}}\right)$ would require that

$\frac{P_{\mathrm{pui}, 2}\left(r_{\mathrm{sh}}\right)}{\epsilon_{\mathrm{kin}}\left(r_{\mathrm{sh}}\right)}=2 \xi\left(r_{\mathrm{sh}}\right) s^{2}\left(\frac{r_{\mathrm{sh}}}{r_{\mathrm{E}}}\right)^{1 / 2} \ln \left(150 \frac{r_{\mathrm{E}}}{r_{\mathrm{sh}}}\right)$

with $\xi\left(r_{\mathrm{sh}}\right) \simeq 0.2$ denoting the PUI abundance at the shock. This would mean that the downstream thermal energy of the PUIs is much higher than the kinetic energy of the upstream solar wind which is forbidden by physical reasons. This again leaves to conclude that PUIs cannot exist in pressure equilibrium with the compressional magnetosonic turbulence that was assumed by Fisk \& Gloeckler (2007) and cannot be responsible for the stochastic particle acceleration up to regions near the solar wind termination shock. 
Requiring that the downstream thermal energy of the PUIs stays below the upstream kinetic energy of the PUIs would require instead an upper border $v_{\infty}$ of the PUI power spectrum defined by

$$
\begin{aligned}
P_{\text {pui }, 2} & =\frac{m s^{2}}{2} \int_{v_{0}}^{v_{\infty}} f_{\text {pui }, 1}(v) v^{4} \mathrm{~d} v \\
& =s^{2}\left[4 \pi f_{\text {pui }} \frac{m}{2} v_{0}^{5} \ln \left(\psi_{\infty}\right)\right] \leq \frac{m}{2} U^{2}\left(n_{\mathrm{s}}+n_{\text {pui }}\right)
\end{aligned}
$$

yielding

$\ln \left(\psi_{\infty}\right) \leq \frac{U^{2}\left(n_{\mathrm{s}}+n_{\text {pui }}\right)}{s^{2} 4 \pi f_{\text {pui }} v_{0}^{5}}=\frac{U^{2}\left(n_{\mathrm{s}}+n_{\text {pui }}\right)}{2 s^{2} n_{\text {pui }} v_{0}^{2}}=\frac{25}{2 s^{2} \xi_{\text {pui }}}\left(\frac{r_{\text {sh }}}{r_{\mathrm{E}}}\right)^{-1 / 2}$.

Evaluating this formula with $s=2.5, \xi_{\text {pui }}=0.2$, and $r_{\mathrm{sh}}=$ $100 r_{\mathrm{E}}$ leads to the result

$\ln \left(\psi_{\infty}\right) \leq \frac{25}{2 \cdot 2.5^{2} \cdot 0.2 \cdot 10}=1$

and means that $\psi_{\infty}=\exp (1)=2.72$ and $v_{\infty} \simeq 2.72 U$, i.e. much lower than required for pressure equilibrium conditions.

\section{The relative effectiveness of energy diffusion and convective changes at ion phase-space transport}

In the following we want to clarify the role of energy diffusion in determining the shape of the PUI-distribution function. We start from the transport equation adequate to describe the phase-space behavior of the PUIs by a distribution function $f(t, r, v)$ (see e.g. Isenberg 1987; Chalov \& Fahr 1996)

$$
\begin{aligned}
\frac{\partial f}{\partial t}+\boldsymbol{U} \frac{\partial f}{\partial \boldsymbol{r}}-\left(\frac{v}{3}\right)\left(\frac{\partial f}{\partial v}\right) \operatorname{div} \boldsymbol{U}= & \frac{1}{v^{2}} \frac{\partial}{\partial v}\left(v^{2} D_{v v} \frac{\partial f}{\partial v}\right) \\
& +Q(\boldsymbol{r}, v)+S(\boldsymbol{r}, v),
\end{aligned}
$$

where $\boldsymbol{U}$ is the solar wind bulk speed, $D_{v v}$ is the velocity diffusion coefficient, and, $Q(\boldsymbol{r}, v)$ and $S(\boldsymbol{r}, v)$ are functions describing PUI-injection sources and -phase-space losses. Terms on the left-hand side of the above Eq. (39) under steady state conditions induce changes with a typical convection time $\tau_{\text {conv }}$ given by

$\tau_{\text {conv }} \approx r / U$,

where $r$ is of the order of the heliocentric distance.

Considering quasi-linear velocity diffusion of particles due to Fermi-2 type interactions with the Alfvénic- or magnetosonic turbulence, both of which are leading to analogous expressions (see Toptygin 1985; Le Roux \& Ptuskin 1998; Chalov \& Fahr 2000). For our estimates here, one can use the diffusion coefficient derived by Schlickeiser (1989) which for estimate purposes can be represented in the following form:

$D_{v v} \approx \delta_{\mathrm{M} 0} \Omega v_{\mathrm{A}}^{2}\left(\frac{k_{0}}{k_{\mathrm{res}}}\right)^{\alpha-1}$,

where $k_{\text {res }}=\Omega / v$ is the resonant wave number, $\Omega$ the proton cyclotron frequency, $v_{\mathrm{A}}$ the Alfvén speed, $k_{0}$ the turbulence outer scale, $\alpha \simeq 5 / 3$ (or $\simeq 3 / 2$ ) the power exponent of the $1 \mathrm{D}$ turbulence spectrum, and $\delta_{\mathrm{M} 0}$ is the fractional turbulence level

$\delta_{\mathrm{M} 0}=\frac{\left\langle\delta \boldsymbol{B}^{2}\right\rangle}{\langle\boldsymbol{B}\rangle^{2}}$, where $\boldsymbol{B}$ is the induction of local interplanetary magnetic field. Typical velocity diffusion times $\tau_{\text {diff }}$ characterizing the action of the first term of the right-hand side of Eq. (39) in changing the distribution function $f$ can be defined by

$\tau_{\mathrm{diff}}^{-1} \approx D_{v v} v^{-2}$.

Combining the relations given by Eqs. (40), (42), (41), and (43) yields as a typical ratio of the characteristic times

$\chi(v, r)=\frac{\tau_{\text {conv }}}{\tau_{\text {diff }}} \approx \delta_{\mathrm{M} 0}\left(\frac{v_{\mathrm{A}}^{2}}{v U}\right)\left(k_{\text {res }} r\right)\left(\frac{k_{0}}{k_{\text {res }}}\right)^{\alpha-1} \sim v^{\alpha-3}$.

At heliocentric distances $r \approx r_{\mathrm{E}}=1 \mathrm{AU}$, one can assume (Chashei et al. 2003) $\delta_{\mathrm{M} 0} \approx 0.1, v_{\mathrm{A}} \approx 0.1 U, k_{0} \approx 10^{-11} \mathrm{~cm}^{-1}$, $\alpha=3 / 2$ for Iroshnikov-Kraichnan turbulence (i.e. a power-law for the spectral energy density $E_{k} \sim k^{-3 / 2}$ ) or $\alpha=5 / 3$ for Kolmogorov turbulence (i.e. $E_{k} \sim k^{-5 / 3}$ ), which are the two mostly found and discussed spectral energy distributions in solar wind turbulence (Bale et al. 2005; Horbury et al. 2005). Then near $1 \mathrm{AU}$, we find a ratio $\chi(U, 1 \mathrm{AU}) \approx 1$ showing that convection and diffusion processes here are of comparable importance for the particles with $v \approx U$, while convection effects are of increasing importance for particles with higher velocities, i.e. with $v>U$. This result is in good agreement with other considerations (Matthaeus \& Goldstein 1986; Jokipii \& Kota 1989; Zank \& Matthaeus 1992; Zank et al. 1996; Horbury \& Balogh 2001). Isenberg (2005), however, concludes that the effect of energy diffusion is small for PUIs. His similar treatment is parametrized by the percentage of turbulence occurring in slab Alfvénic fluctuations. Nevertheless, these two approaches can be conciliated if this percentage is assumed to amount about $20 \%$.

At larger heliocentric distances, $r>1 \mathrm{AU}$, the frozen-in Parker magnetic field near the ecliptic is nearly azimuthal and decreases with $(1 / r)$. Correspondingly, the resonant wavevector behaves like $k_{\text {res }} \sim \Omega \sim\langle B\rangle \sim r^{-1}$, which leads to $k_{\text {res }} r=$ const. Besides that, as argued by Chashei et al. (2003), the following $r$-dependences can be expected: $k_{0} \sim r^{-1}$ and $\delta_{\mathrm{M} 0} \sim r^{-2 / 3}$. Consequently, the radial dependence of $\chi(v, r)$ is exclusively determined by the $r$-dependence of $\delta_{\mathrm{M} 0}$ meaning that

$\chi(v, r) \approx\left(\frac{r}{r_{\mathrm{E}}}\right)^{-2 / 3}\left(\frac{v}{U}\right)^{-7 / 3}$

in case of Kolmogorov turbulence. The above Eq. (45) shows that at larger solar distances energy diffusion of particles cannot determine the shape of the suprathermal tail of the resulting PUIdistribution function.

In fact, assuming for reasons of a better clarification, just to the contrast here, a dominance of the energy diffusion, i.e. neglecting all the terms in Eq. (39) except for the diffusion term, would deliver as a result of the transport equation the distribution $f \sim v^{-\alpha}$. This distribution thus should evidently be much flatter than the obviously observed distribution $f \sim v^{-5}$ (Fisk \& Gloeckler 2006) indicating that energy diffusion in fact plays an inferior role.

This result also turned out from more quantitative calculations of wave-particle interactions in the outer heliosphere by Chalov et al. (2006a) or Fahr \& Chashei (2007) in which a consistent treatment of PUI transport in phase-space and wave turbulence transport in $k$-space was presented. From these calculations it becomes evident that in fact in the outer heliosphere PUIs drive turbulence powers, but are not efficiently enough profiting from energy diffusion to produce power-law tails.

The only way to fill up ion tails at energies larger than $1 \mathrm{keV}$ resulting in some power-law distribution, as it seems to us, is 
to inject ions into the PUI regime at higher energies of about $\geq 50 \mathrm{keV}$ and then let them cool to smaller energies at their coconvection with the solar wind. This would be a process similar to the one of PUIs originating from freshly ionized neutral atoms which are injected at about $1 \mathrm{keV}$ (see Fahr 2007; Siewert \& Fahr 2008). In the following, we study such a high-energy injection mechanism to the PUI energy regime resulting from ACR ions that are adiabatically cooled to lower energies and, therefore, can be called ACR-PUIs.

\section{Injection to the PUI regime from the high-energy side}

Here we first want to study the modulated part of the ACRs with origin near the solar wind termination shock and investigate whether they can perhaps serve as a possible injection seed from high energies into the PUI regime. We start from the analytic expression for the modulated ACR spectral intensity given for the case of a spherically symmetric solar modulation. The differential ACR intensity for this case is given by the following expression (Stawicki et al. 2000):

$$
\begin{aligned}
j(r, p)= & p^{2} f(r, p) \\
= & \frac{3}{b} \int \mathrm{d} r_{0} \int \mathrm{d} p_{0} \frac{S\left(r_{0}, p_{0}\right)}{U} \frac{p_{0} y_{0}}{\phi}\left(\frac{r_{0}}{r}\right)^{\frac{1+\delta}{2}}\left(\frac{p_{0}}{p}\right)^{\frac{3 \delta-4 \gamma-5}{2(2+\gamma)}} \\
& \times \exp \left(-\frac{y_{0}\left(1+h^{2}\right)}{\phi}\right) I_{\frac{1+\delta}{1+\gamma-\delta}}\left(\frac{2 y_{0} h}{\phi}\right),
\end{aligned}
$$

where $S\left(r_{0}, p_{0}\right)$ denotes the ACR source function with source coordinates $r_{0}$ and $p_{0}, I_{\mathrm{n}}$ is the modified Bessel function of the first kind, $U$ denotes the solar wind bulk velocity, and $h$ and $\phi$ have the following definitions:

$h=\left(\frac{r}{r_{0}}\right)^{\frac{1+\gamma-\delta}{2}}\left(\frac{p}{p_{0}}\right)^{\frac{3}{2 b}}$,

$\phi=1-\left(\frac{p}{p_{0}}\right)^{\frac{3 v}{2+\gamma}}$

with the following additional quantities

$y_{0}=\frac{v}{(1+\gamma-\delta)^{2}} \frac{r_{0} U}{\kappa_{r r, 0}}$,

$b=\frac{2+\gamma}{(1+\gamma-\delta)}$

$v=\left(1+\gamma-\delta+\frac{2+\gamma}{3} \epsilon\right)$

and the following assumptions of the $r$ - and $p$-dependences:

$U(r) \sim r^{\gamma}, \quad \kappa_{r r}(r, p)=\kappa_{r r, 0}\left(\frac{r}{r_{\mathrm{E}}}\right)^{\delta}\left(\frac{p}{p_{\mathrm{E}}}\right)^{\epsilon}$

with an arbitrary reference momentum $p_{\mathrm{R}}$ for the bulk velocity and the spatial diffusion coefficient $\kappa_{r r}$.

As shown by Stawicki et al. (2000), the above expression can be simplified for low values of ACR particle momenta $p$ and then yields the following expression for the ACR distribution:

$f(r, p) \simeq \frac{3}{b \Gamma(b)} \int \mathrm{d} r_{0} \int \mathrm{d} p_{0} \frac{S\left(r_{0}, p_{0}\right)}{p_{0} U} y_{0}^{b} \exp \left(-y_{0}\right)$.
The modulation of the ACR spectrum due to spatial diffusion is contained in $y_{0}$. This shows that, hence, the ansatz by Stawicki et al. (2000) is an analytical way to express the modulated distribution function of the ACRs in the heliosphere. In the frame of its accuracy, this approach yields a valuable way to take the modulated ACR spectrum as the source of our cooling mechanism. The spectrum of the ACR particles at the solar wind termination shock serves as the ACR source function and, thus with good reasons, one adopts the function developed by Drury (1983) for an ideally planar shock which is given by

$$
\begin{aligned}
& S\left(r_{0}, p_{0}\right)=\frac{1}{r_{0}^{2}} \frac{\partial}{\partial r_{0}} r_{0}^{2} \zeta_{0} \\
& =\frac{1}{r_{0}^{2}} \frac{\partial}{\partial r_{0}} r_{0}^{2}\left[S_{\mathrm{ACR}, 0} \cdot\left(\frac{p_{0}}{p_{\mathrm{i}}}\right)^{-q} \cdot \exp \left(-\frac{p_{0}}{p_{\max }}\right) \delta\left(r_{0}-r_{\mathrm{sh}}\right)\right],
\end{aligned}
$$

where the spectral index $q$ is connected with the shock compression ratio $s$ by $q=3 s /(s-1) . p_{i}, p_{\max }$ are appropriate values of the Fermi-1 injection momentum and of the upper cut-off momentum, respectively. This then leads to

$$
\begin{aligned}
f(r, p) \simeq & \frac{3 S_{\mathrm{ACR}, 0}}{b \Gamma(b) U} \int_{0}^{\infty} \frac{\mathrm{d} p_{0}}{p_{\mathrm{i}}}\left(\frac{p_{0}}{p_{\mathrm{i}}}\right)^{-q-1} \exp \left(-\frac{p_{0}}{p_{\max }}\right) \\
& \times\left(\frac{v}{(1+\gamma-\delta)^{2}} \frac{r_{\mathrm{sh}} U}{\kappa_{r r, \mathrm{sh}}}\right)^{b} \exp \left(-\frac{v}{(1+\gamma-\delta)^{2}} \frac{r_{\mathrm{sh}} U}{\kappa_{r r}, \mathrm{sh}}\right) .
\end{aligned}
$$

This expression represents a constant distribution function. Numerical simulations by Fichtner \& Sreenivasan (1999) show a linear dependence of the ACR flux on the energy $\left(j_{E} \sim E\right)$. This means that the distribution function in phase-space is constant which can be shown by considerations about normalization of the differential flux and the distribution function:

$f=4 \pi \frac{m j_{E}}{p^{3}} \frac{\mathrm{d} E}{\mathrm{~d} p} \sim \frac{j_{E}}{E}=$ const.

Therefore, the $f(r, p)$ of the ACR particles is given by a constant value which follows from modulation theory:

$f(r, p) \simeq f_{\text {const }}$.

From the above expression we develop the total streaming $S\left(r, p_{\text {pui, }}\right)$ (see Gleeson \& Axford 1967; Fahr 1990; Fahr \& Verscharen 2009) for the ACR-PUIs in the range between the injection momentum $p_{\mathrm{i}}$ and an upper border $p_{\text {pui,i }}$. ACR particles with higher momenta propagate only according to the transport equation for cosmic rays and do not participate in the diffusive injection. With these considerations we find

$S=\int_{p_{\mathrm{i}}}^{p_{\text {pui,i }}} p^{2} U\left(\frac{1}{3} f-\frac{1}{3 p} \frac{\partial}{\partial p} f\right) \mathrm{d} p=\frac{\left(p_{\text {pui, }}^{3}-p_{\mathrm{i}}^{3}\right)}{9} U f_{\text {const }}$

as the total particle streaming at each place $r$. Since we expect that $p_{\text {pui, }} \gg p_{\mathrm{i}}$, the lower border $p_{\mathrm{i}}$ can be neglected in Eq. (58).

Now we consider the local spatial divergence of this ACRPUI streaming at some lower momentum border and take this to be the high-energy injection source $Q$ to the PUI regime. With this choice, we obtain

$Q=\frac{1}{r^{2}} \frac{\partial}{\partial r} r^{2} S=\frac{2 p_{\text {pui, }}^{3} U f_{\text {const }}}{9 r}$

as the divergence of the ACR particle flux, constituting a PUI source. 


\section{PUI Boltzmann-Vlasov equation with ACR-induced injection}

In the "solar" rest frame (SF), the representative BoltzmannVlasov equation (BVE) is given by

$\left(\boldsymbol{U} \cdot \nabla_{r}\right) f+\left(\left.\frac{\mathrm{d} v}{\mathrm{~d} t}\right|_{\mathrm{m}} \cdot \nabla_{v}\right) f=U \frac{\partial f}{\partial r}+\left.U \frac{\partial v}{\partial r}\right|_{\mathrm{m}} \frac{\partial f}{\partial v}=P(r, v)$

under the prevailing conditions in the outer heliosphere, i.e. negligible energy diffusion, for the stationary case (Fahr 2007). The corresponding BVE equation in the "solar wind" rest frame (WF) has the form (Fahr 2007)

$\frac{\partial \tilde{f}}{\partial t}+\frac{1}{v^{2}} \frac{\partial}{\partial v}\left(v^{2} \dot{v}_{\mathrm{m}} \tilde{f}\right)=\widetilde{P}(t, v)$,

where the second term on the left-hand side describes the velocity-space divergence of the phase-space flow connected with the magnetically induced deceleration (i.e. magnetic cooling), which is indicated by the subscript $\mathrm{m}$. The coordinate $t=t(r)$ denotes the proper time in the co-moving reference frame (WF). Furthermore, $\widetilde{P}(r, v)$ is the local ion injection rate to the PUI regime.

For low energies $(\leq 1 \mathrm{keV})$, this rate is due to locally freshly ionized neutral $\mathrm{H}$-atoms and is given by $\widetilde{P}(r, v)=\beta(r) \frac{1}{4 \pi v^{2} U}$ $\delta(v-U)$ with $\beta(r)$ being the local PUI production rate (Fahr \& Siewert 2008).

For high energies ( $\geq 50 \mathrm{keV}$ for the ACR-PUIs), the relevant injection, in contrast to the normal sub-keV PUIs, in our view is given by the upper expression derived from the modulated ACR spectrum and given by Eq. (59).

Furthermore, $\dot{v}_{\mathrm{m}}$ is determined by the magnetically induced velocity decrease of particles with a velocity $v$, when they are convected outwards with the solar wind bulk flow at a mean velocity $U$ to larger distances where the co-convected interplanetary magnetic field $B$ appears reduced in magnitude (Fahr 2007). At larger distances $r \geq 5$ AU near the ecliptic, the magnetic field decreases like $(1 / r)$ (i.e. in case of the nearly azimuthal, distant Parker field). Under these conditions, one finds the following $v$-dependent magnetic velocity-space drift

$\dot{v}_{\mathrm{m}}=-U \frac{v}{r}$

and its associated radial gradient

$\frac{\partial v_{\mathrm{m}}}{\partial r}=\frac{1}{U} \dot{v}_{\mathrm{m}}=-\frac{v}{r}$

in the form given by Fahr \& Siewert (2008).

Ions which are picked up at $r_{v}$ with a velocity $U$ will, without other processes being involved, have "magnetically" cooled down to a velocity $v$ at $r$ if the relation $r_{v}(v)=r v / U$ is fulfilled. The injection of freshly created PUIs at $r_{v}(v)$ with an initial velocity $v=U$ will be responsible for ions with velocity $v$ at $r$.

Taking all these constraints together, one finally finds, when reminding that the time and distance coordinates are related to each other by $\mathrm{d} r=U \mathrm{~d} t$, that for velocities $v \leq U$ the solution for $\tilde{f}$ in the WF is given by (see Fahr 2007; Siewert \& Fahr 2008)

$\tilde{f}_{\leq}=\frac{1}{2 \pi} \frac{r \beta\left(\frac{v}{U} r\right)}{U} v^{-3}$.

This distribution function $\tilde{f}_{\leq}$can be evaluated for larger solar distances $r \geq r_{0}=5 \mathrm{AU}$ in the upwind hemisphere for nearecliptic positions assuming that at such solar distances the upwind $\mathrm{H}$-atom density can be considered as essentially constant, meaning that $n_{\mathrm{H}}\left(\frac{v}{U} r\right)=n_{\mathrm{H}}(r)=n_{\mathrm{H}, \infty}$. This in fact is an acceptable approximation for solar distances $r \geq 5 \mathrm{AU}$ and velocities $1 U \geq v \geq 0.2 U$, and then leads to

$$
\tilde{f}_{\leq}=\frac{r}{2 \pi U} v_{\mathrm{ex}, \mathrm{E}} r_{\mathrm{E}}^{2}\left(\frac{v}{U} r\right)^{-2} n_{\mathrm{H}, \infty} v^{-3}=\frac{v_{\mathrm{ex}, \mathrm{E}} r_{\mathrm{E}}^{2} U}{2 \pi r} n_{\mathrm{H}, \infty, v^{-5}}
$$

i.e. to the astonishing fact that under pure magnetic cooling, the resulting PUI-distribution function for velocities $v \leq 1 U$ is a power-law with the interesting power-index $\alpha=-5$ predicted and confirmed by Fisk \& Gloeckler (2006, 2007); however, in their case expected as result of an assumed quasi-equilibrium state established between magnetoacoustically driven ion energy diffusion and magnetoacoustic turbulence generation.

The above result is valid only for ions with $v \leq U$. One possibility that we now start to see here is that for ions with velocities $v \geq 1 U$, i.e. much higher than the original PUI-injection threshold, one has to consider in addition some high-energy injection rate due to modulated ACRs as we have derived above.

For those ACR-induced ions (i.e. for the ACR-PUIs) with the relevant source function

$Q=\frac{2 p_{\text {pui,i }}^{3} U f_{\text {const }}}{9 r}$,

we find analogously to Eq. (64) for ACR-PUIs

$\tilde{f}_{\geq}=\frac{1}{2 \pi} \frac{r Q\left(r \frac{v}{v_{\text {pui, }}}\right)}{U} v^{-3}=\frac{1}{2 \pi} \frac{r \cdot\left[\frac{2 p_{\text {pui, }}^{3} \frac{v_{\text {pui,i }}}{v}}{9 r} U f_{\text {const }}\right]}{U} v^{-3}$,

which finally leads to an $r$-independent distribution function for the range $v \geq U$ in the form

$\tilde{f}_{\geq}=\frac{1}{9 \pi} m_{\mathrm{p}}^{3} v_{\text {pui, }}^{4} f_{\text {const }} v^{-4}$

Altogether, thus, we obtain the total PUI distribution in the following form:

$$
\begin{aligned}
\tilde{f} & =\tilde{f}_{\leq}+\tilde{f}_{\geq} \\
& =\frac{v_{\text {ex }, \mathrm{E}} r_{\mathrm{E}}^{2} U}{2 \pi r} n_{\mathrm{H}, \infty} v^{-5} H(U-v)+\frac{1}{9 \pi} m_{\mathrm{p}}^{3} v_{\text {pui, } \mathrm{i}}^{4} f_{\text {const }} v^{-4},
\end{aligned}
$$

where $H(x)$ is the well-known step function with $H(x \leq 0)=0$ and $H(x \geq 0)=1$.

In any case, the above result shows that a velocity-power index of " -5 " is obtained for the velocity range $v \leq U \leq v_{\text {pui,i }}$ and a power-index of " -4 " is obtained for the velocity range $U \leq v \leq v_{\text {pui,i }}$ where $v_{\text {pui, }}=p_{\text {pui, }} / m_{\mathrm{p}}$.

Rescaling velocity in units of $U$ (i.e. $X=v / U$ ) leads to the total distribution function

$$
\begin{aligned}
\tilde{f} & =\frac{\left(\sigma_{\mathrm{ex}} n_{\mathrm{sE}} U\right) r_{\mathrm{E}}^{2}}{2 \pi r U^{4}} n_{\mathrm{H}, \infty} X^{-5} H(1-X)+\frac{1}{9 \pi} m_{\mathrm{p}}^{3} X_{\mathrm{pui}, \mathrm{i}}^{4} f_{\mathrm{const}} X^{-4} \\
& =\frac{n_{\mathrm{sE}}}{2 \pi U^{3}}\left[\Lambda\left(\frac{r_{\mathrm{E}}}{r}\right) X^{-5} H(1-X)+\frac{2}{9 \pi} \frac{m_{\mathrm{p}}^{3} X_{\mathrm{pui}, \mathrm{i}}^{4} U^{3} f_{\text {const }}}{n_{\mathrm{s}, \mathrm{E}}} X^{-4}\right]
\end{aligned}
$$

We introduce now two dimensionless characterizing quantities. First, we define

$\Lambda=\left(\sigma_{\mathrm{ex}} n_{\mathrm{H}, \infty} r_{\mathrm{E}}\right) \simeq 2.3 \times 10^{-3}$,

where the charge exchange cross section $\sigma_{\mathrm{ex}} \simeq 10^{-15} \mathrm{~cm}^{2}(\mathrm{Fahr}$ 1971 ) and the hydrogen density $n_{\mathrm{H}, \infty} \simeq 0.15 \mathrm{~cm}^{-3}$ (Izmodenov et al. 2003) are used. 
With

$\Delta=\frac{2}{9 \pi} \frac{m_{\mathrm{p}}^{3} X_{\text {pui,i }}^{4} U^{3} f_{\text {const }}}{n_{\mathrm{s}, \mathrm{E}}}$,

one can finally find

$\tilde{f}(r, v)=\frac{n_{\mathrm{SE}}}{2 \pi U^{3}}\left[\Lambda\left(\frac{r_{\mathrm{E}}}{r}\right) \cdot X^{-5} H(1-X)+\Delta \cdot X^{-4}\right]$.

Measurements by the SWICS instrument on the ULYSSES space probe show a PUI-phase-space density of about $100 \mathrm{~s}^{3} \mathrm{~km}^{-6}$ at two times the solar wind velocity at a distance of 5.26 AU (Gloeckler 2003). Equation (73) leads to a value for the PUI-phase-space density of about $\tilde{f}_{\leq} \simeq 155 \mathrm{~s}^{3} \mathrm{~km}^{-6}$, which is in good accordance to the observations. The difference could be a consequence of the assumption of a constant $n_{\mathrm{H}}$, especially at small distances from the sun.

Observations by the LECP instruments on VOYAGER 1 and 2 (Lanzerotti et al. 2001) show an $r$-independent proton intensity in the energy range $0.57-1.78 \mathrm{MeV}$ of about $j_{E} \simeq 4 \times 10^{-4}$ particles per $\left(\mathrm{cm}^{2} \mathrm{~s} \mathrm{sr} \mathrm{MeV}\right)$, which corresponds to $j_{E} \simeq 250$ particles per $\left(\mathrm{cm}^{2} \mathrm{~s}\right.$ sr erg) in Gaussian units. This value can be converted to a phase-space density (cf. Eq. (56)):

$\tilde{f}_{\geq}=4 \pi \frac{m_{\mathrm{p}}^{3} j_{E}}{p^{2}(1 \mathrm{MeV})}=2.7 \times 10^{-39} \mathrm{~s}^{3} \mathrm{~cm}^{-6}$.

If we assume that these ions observed by Lanzerotti et al. (2001) are ACR-PUI particles with the proposed behavior, this leads to a value for $\Delta$ obtained from the measurements of

$\Delta=\frac{2 \pi U^{3}}{n_{\mathrm{sE}}} X^{4}(1 \mathrm{MeV}) \tilde{f}_{\geq}=3.1 \times 10^{-10}$.

We take this observational value to avoid uncertainties at the determination of the ACR intensity $f_{\text {const }}$ and the injection border $X_{\text {pui,i }}$. The calculated spectra are shown in Fig. 1.

\section{Comparison of our results with data}

Now we want to compare the above results with VOYAGER and ULYSSES spectral ion data. For that purpose, we first transform the above velocity distribution function into a distribution of spectral energy flux $j(E)$ given in units of (ions/( $\left.\mathrm{cm}^{2} \mathrm{~s} \mathrm{sr} \mathrm{MeV}\right)$ ). This then leads to

$$
\begin{aligned}
j(E) & \sim \sqrt{E} \cdot f(E) \sim \sqrt{E}\left(\frac{E}{E_{0}}\right)^{\left(1-\alpha_{v}\right) / 2} \\
& \sim\left(\frac{E}{E_{0}}\right)^{\left(2-\alpha_{\nu}\right) / 2} \sim\left(\frac{E}{E_{0}}\right)^{-\alpha_{E}},
\end{aligned}
$$

where $\alpha_{v}$ and $\alpha_{E}$ are the velocity- and the energy-power indices, respectively. As one can easily see, $\alpha_{v}=5$ leads to $\alpha_{E}=1.5$, whereas $\alpha_{v}=4$ would lead to $\alpha_{E}=1.0$. Looking into the data obtained by VOYAGER 1 during the year 2004 (see Decker et al. 2005,2006 ) before the shock crossing occurred, one can find even though the data are very time-variable in this interval - that for ions with higher energy $\left(E \simeq 10^{3} \mathrm{keV}\right)$ the energy-power index has been observed with $\alpha_{E}(E \geq 200 \mathrm{keV}) \simeq 1.5$, whereas interestingly enough for ions with lower energy (i.e. $E \simeq 40 \mathrm{keV}$ ) a smaller power-index of $\alpha_{E}(E \leq 200 \mathrm{keV}) \simeq 1.0$ seems to be indicated. That supports our above derived theoretical prediction that the velocity-power index of ACR-induced PUIs in the outer heliosphere is $\alpha_{v} \simeq 4$, rather than $\alpha_{v} \simeq 5$ as for the ions with higher energy $(\mathrm{MeV})$.

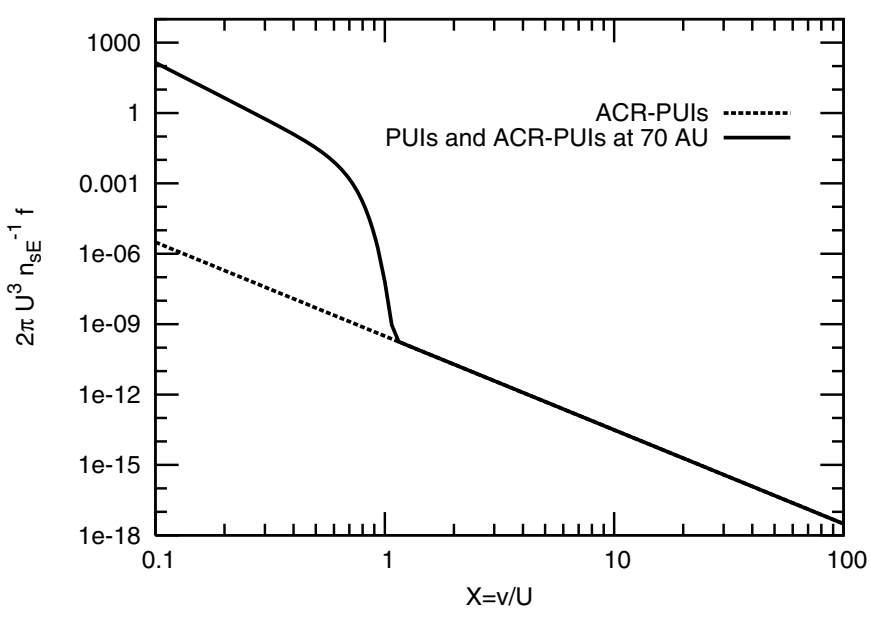

Fig. 1. Phase-space density spectra for PUIs and ACR-PUIs. The PUI distribution is dependent on the distance from sun, whereas the ACRPUIs are not. PUIs cannot gain velocities above $U$, i.e. $X=1$. The solid line is led down to the ACR-PUI spectrum at the cut-off at $X=1$ artificially.

Also during the most recent VOYAGER 2 crossing of the shock (see Decker et al. 2008) it became evident that the energypower indices registered both before and after the shock crossing show values of $\alpha_{E, 1} \simeq \alpha_{E, 2} \simeq 1.2 \pm 0.2$ which also nicely confirms a velocity index of $\alpha_{v} \simeq 4$.

In addition, it is important to recognize that the particle detectors of the VOYAGER spacecraft did not see radial changes of the spectral flux intensity $j(E)$ at the lower energy channels (0.35-1.5 MeV) during the period from years 1995 through 2005 (Lanzerotti et al. 2001; Decker \& Krimigis 2003; Krimigis et al. 2003). This is also a support for the newly derived theoretical expression given in Eq. (73) and showing that a distanceindependent intensity can be expected at energies $5 \mathrm{keV} \leq E \leq$ $100 \mathrm{keV}$. Only the part of the spectrum that is a result of cooled PUIs shows a dependence on the distance due to the different PUI abundances. The ACR-PUIs that are injected from ACRs and cooled from high energies, however, are proven to have no radial variation.

Our model explains occurring power-law tails with an index -4 . There are, however, observations around $5 \mathrm{AU}$ undoubtedly showing a power-index -5 for suprathermal ion tails (e.g. Gloeckler 2003). As we can show, the favored explanation by Fisk \& Gloeckler $(2006,2007)$ cannot hold in the described way. Maybe other processes (such as anomalous Fermi-2 type waveparticle energy diffusion), which are not treated in our approach, can lead to a power-index of -5 at energies just above $5 \mathrm{keV}$. But this interaction does not explain the occurrence of extended power-law tails at larger distances (Chalov et al. 2004).

It is perhaps still a little bit an open question, where, i.e. at what lower energy, one should cut off the modulated ACR spectrum to calculate the injection to the PUI regime. Even though this does not count very much in quantitative terms, since the lower energy part of the modulated ACR spectrum has in fact a constant distribution function (see Eq. (56)), it may nevertheless represent an intellectually interesting question, to decide up to what energies convection and Fermi-2 energy diffusion are dominant, and from what energies upwards Fermi- 1 acceleration and spatial diffusion dominate. There is one clear hint given to answer this question, namely by the momentum dependence of 
the spatial diffusion coefficient $\kappa_{r r}(r, p)$ generally given in the form

$\kappa(r, p)=\kappa_{0} \kappa_{r}(r) \kappa_{p}(p) \sim\left(\frac{r}{r_{\mathrm{E}}}\right)^{\delta}\left(\frac{p}{p_{\mathrm{R}}}\right)^{\epsilon}$,

where, dependent on the momentum range, the exponent $\gamma$ is expected to be in the range $1 \leq \gamma \leq 2$ (see Le Roux \& Potgieter 1992; Jokipii 1996). This shows that spatial diffusion becomes less and less efficient, the lower the ion momentum $p$ is. Below some critical value $p_{\mathrm{c}}$, ions lose their degree of kinetic freedom to spatially diffuse relative to the solar wind background flow and, thus, they are simply convected outwards with the solar wind bulk flow then.

The determination of the absolute height of the ACR-PUI spectrum is a slightly problematic endeavor. In our calculation, we normalize the absolute spectrum with the aid of observational data. The ACR intensity $f_{\text {const }}$ and the limit for the momentum, up to which the injection is effective due to the total streaming (see Eq. (58)), are quite uncertain. Another open question results from a lack of data. Especially, the transition from the PUI to the ACR-PUI part has not been covered sufficiently by particle detectors in the outer parts of the heliosphere. Maybe future missions can provide a closer look to this part of the spectrum.

Acknowledgements. H.-J. F. and D. V. are grateful to the Deutsche Forschungsgemeinschaft for financial support within the frame of the DFG project Fa 97/31-2. One of us, I. V. C., is grateful for financial support in the frame of DFG project 436 RUS113/110/0-4.

\section{References}

Bale, S. D., Kellogg, P. J., Mozer, F. S., Horbury, T. S., \& Reme, H. 2005, Phys. Rev. Lett., 94, 215002

Bogdan, T. J., Lee, M. A., \& Schneider, P. 1991, J. Geophys. Res., 96, 161

Bzowski, M., Möbius, E., Tarnopolski, S., Izmodenov, V., \& Gloeckler, G. 2008, A\&A, 491, 7

Chalov, S. V., \& Fahr, H. J. 1996, Sol. Phys., 168, 389

Chalov, S. V., \& Fahr, H. J. 1998, A\&A, 335, 746

Chalov, S. V., \& Fahr, H. J. 1999a, Ap\&SS, 264, 509

Chalov, S. V., \& Fahr, H. J. 1999b, Sol. Phys., 187, 123

Chalov, S. V., \& Fahr, H. J. 2000, A\&A, 360, 381

Chalov, S. V., Fahr, H. J., \& Izmodenov, V. V. 2003, J. Geophys. Res., 108, 1266

Chalov, S. V., Alexashov, D. B., \& Fahr, H. J. 2004, A\&A, 416, L31

Chalov, S. V., Alexashov, D. B., \& Fahr, H.-J. 2006a, Astrophys. Space Sci. Trans., 2, 19

Chalov, S. V., Alexashov, D. B., \& Fahr, H. J. 2006b, Astron. Lett., 32, 206

Chashei, I. V., Fahr, H. J., \& Lay, G. 2003, Adv. Space Res., 32, 507

Decker, R. B., \& Krimigis, S. M. 2003, Adv. Space Res., 32, 597

Decker, R. B., Krimigis, S. M., Roelof, E. C., et al. 2005, Sci., 309, 2020

Decker, R. B., Roelof, E. C., Krimigis, S. M., \& Hill, M. E. 2006, in Physics of the Inner Heliosheath, ed. J. Heerikhuisen, V. Florinski, G. P. Zank, \& N. V. Pogorelov, AIP Conf. Ser., 858, 73

Decker, R. B., Krimigis, S. M., Roelof, E. C., et al. 2008, Nature, 454, 67

Drury, L. O. 1983, Rep. Prog. Phys., 46, 973
Fahr, H. J. 1971, A\&A, 14, 263

Fahr, H. J. 1990, in Physics of the Outer Heliosphere, ed. S. Grzedzielski, \& D. E. Page, 327

Fahr, H.-J. 2007, Ann. Geophys., 25, 2649

Fahr, H., \& Chashei, I. 2002a, in COSPAR, Plenary Meeting, Vol. 34, 34th COSPAR Scientific Assembly

Fahr, H. J. \& Chashei, I. V. 2002b, A\&A, 395, 991

Fahr, H. J., \& Chashei, I. V. 2007, Astron. Astrophys. Trans., 26, 621

Fahr, H. J., \& Lay, G. 2000, A\&A, 356, 327

Fahr, H. J., \& Rucinski, D. 1999, A\&A, 350, 1071

Fahr, H.-J., \& Siewert, M. 2008, A\&A, 484, L1

Fahr, H.-J., \& Verscharen, D. 2009, Astrophys. Space Sci. Trans., 5, 21

Fahr, H. J., \& Ziemkiewicz, J. 1988, A\&A, 202, 295

Fichtner, H. 2001, Space Sci. Rev., 95, 639

Fichtner, H., \& Sreenivasan, S. R. 1999, Adv. Space Res., 23, 535

Fichtner, H., Le Roux, J. A., Mall, U., \& Rucinski, D. 1996, A\&A, 314, 650

Fisk, L. A., \& Gloeckler, G. 2006, ApJ, 640, L79

Fisk, L. A., \& Gloeckler, G. 2007, PNAS, 104, 5749

Gleeson, L. J., \& Axford, W. I. 1967, ApJ, 149, L115

Gloeckler, G., 2003, in Solar Wind Ten, ed. M. Velli, R. Bruno, F. Malara, \& B. Bucci, AIP Conf. Ser., 679, 583

Horbury, T. S., \& Balogh, A. 2001, J. Geophys. Res., 106, 15929

Horbury, T. S., Forman, M. A., \& Oughton, S. 2005, Plasma Phys. Contr. F., 47, B703

Isenberg, P. A. 1987, J. Geophys. Res., 92, 1067

Isenberg, P. A. 2005, ApJ, 623, 502

Isenberg, P. A., Smith, C. W., \& Matthaeus, W. H. 2003, ApJ, 592, 564

Izmodenov, V., Gangopadhyay, P., Gruntman, M., \& Judge, D. 2003, in Solar Wind Ten, ed. M. Velli, R. Bruno, F. Malara, \& B. Bucci, AIP Conf. Ser., 679, 198

Jokipii, J. R. 1996, ApJ, 466, L47

Jokipii, J. R., \& Kota, J. 1989, Geophys. Res. Lett., 16, 1

Király, P. 2005, in International Cosmic Ray Conference, Vol. 1, International Cosmic Ray Conference, 339

Krimigis, S. M., Decker, R. B., Hill, M. E., et al. 2003, Nature, 426, 45

Lanzerotti, L. J., Krimigis, S. M., Decker, R. B., et al. 2001, Space Sci. Rev., 97, 243

Lee, M. A. \& Ip, W.-H. 1987, J. Geophys. Res., 92, 11041

Le Roux, J. A., \& Potgieter, M. S. 1992, ApJ, 390, 661

Le Roux, J. A., \& Ptuskin, V. S. 1998, J. Geophys. Res., 103, 4799

Matthaeus, W. H., \& Goldstein, M. L. 1986, Phys. Rev. Lett., 57, 495

Matthaeus, W. H., Dasso, S., Weygand, J. M., et al. 2005, Phys. Rev. Lett., 95, 231101

McDonald, F. B., Stone, E. C., Cummings, A. C., et al. 2003, Nature, 426, 48

Rucinski, D., \& Fahr, H. J. 1991, Ann. Geophys., 9, 102

Rucinski, D., Fahr, H. J., \& Grzedzielski, S. 1993, Planet. Space Sci., 41, 773

Rucinski, D., Bzowski, M., \& Fahr, H. J. 2003, Ann. Geo., 21, 1315

Schlickeiser, R. 1989, ApJ, 336, 243

Siewert, M., \& Fahr, H.-J. 2008, A\&A, 485, 327

Smith, C. W., Matthaeus, W. H., Zank, G. P., et al. 2001, J. Geophys. Res., 106, 8253

Stawicki, O. 2004, J. Geophys. Res., 109, 4105

Stawicki, O., Fichtner, H., \& Schlickeiser, R. 2000, A\&A, 358, 347

Toptygin, I. N. 1985, NASA STI/Recon Technical Report A, 87, 10094

Winske, D., \& Leroy, M. M. 1984, J. Geophys. Res., 89, 2673

Winske, D., Quest, K. B., Tanaka, M., \& Wu, C. S. 1985, J. Geophys. Res., 90, 123

Zank, G. P., \& Matthaeus, W. H. 1992, J. Geophys. Res., 97, 17189

Zank, G. P., Matthaeus, W. H., \& Smith, C. W. 1996, J. Geophys. Res., 101, 17093 